\title{
A MultiSite Gateway ${ }^{\mathrm{TM}}$ vector set for the functional analysis of genes in the model Saccharomyces cerevisiae
}

Astrid Nagels Durand ${ }^{1,2}$, Tessa Moses ${ }^{1,2,3,4}$, Rebecca De Clercq ${ }^{1,2}$, Alain Goossens $s^{1,2^{*}+}$ and Laurens Pauwels $s^{1,2+}$

\begin{abstract}
Background: Recombinatorial cloning using the Gateway ${ }^{\top M}$ technology has been the method of choice for high-throughput omics projects, resulting in the availability of entire ORFeomes in Gateway ${ }^{\text {TM }}$ compatible vectors. The MultiSite Gateway ${ }^{\mathrm{TM}}$ system allows combining multiple genetic fragments such as promoter, ORF and epitope tag in one single reaction. To date, this technology has not been accessible in the yeast Saccharomyces cerevisiae, one of the most widely used experimental systems in molecular biology, due to the lack of appropriate destination vectors.
\end{abstract}

Results: Here, we present a set of three-fragment MultiSite Gateway ${ }^{\mathrm{TM}}$ destination vectors that have been developed for gene expression in S. cerevisiae and that allow the assembly of any promoter, open reading frame, epitope tag arrangement in combination with any of four auxotrophic markers and three distinct replication mechanisms. As an example of its applicability, we used yeast three-hybrid to provide evidence for the assembly of a ternary complex of plant proteins involved in jasmonate signalling and consisting of the JAZ, NINJA and TOPLESS proteins.

Conclusion: Our vectors make MultiSite Gateway ${ }^{\mathrm{TM}}$ cloning accessible in S. cerevisiae and implement a fast and versatile cloning method for the high-throughput functional analysis of (heterologous) proteins in one of the most widely used model organisms for molecular biology research.

Keywords: Gateway cloning, MultiSite, Saccharomyces cerevisiae, Yeast, Vector, Fusion protein, Epitope tag, Jasmonate, Arabidopsis thaliana

\section{Background}

The model organism Saccharomyces cerevisiae has contributed greatly to our current understanding on eukaryotic genes, their products, and their functions. Decades of study have resulted in an extensive knowledge on yeast physiology, genetics, and the molecular functions and interactions of its proteins. Furthermore, this unicellular eukaryotic system is well suited for the study of basic cellular processes which are often conserved in higher eukaryotes. Because of its ease for

\footnotetext{
* Correspondence: algoo@psb.vib-ugent.be

${ }^{\dagger}$ Equal contributors

'Department of Plant Systems Biology, VIB, Technologiepark 927, B-9052,

Gent, Belgium

${ }^{2}$ Department of Plant Biotechnology and Bioinformatics, Ghent University,

Technologiepark 927, B-9052, Ghent, Belgium

Full list of author information is available at the end of the article
}

genetic modification and fast growth, yeast became the system of choice for in vivo protein analyses from other eukaryotes. S. cerevisiae was used, for example, to perform proteome-wide analysis of the human proteinprotein interaction networks [1], to systematically analyze protein-DNA interaction networks of the nematode C. elegans [2], and to produce high-value bioactive plant secondary metabolites through metabolic engineering approaches [3].

Large scale genomics approaches to uncover protein function are adopted more and more in the current era of systems biology research. To cope with the large amounts of constructs needed, scientists make use of high-throughput cloning technologies, such as the Gateway $^{\mathrm{TM}}$ technology (Invitrogen; http://www.invitrogen. $\mathrm{com} /$ ), which is based on the site-specific recombination system from the bacteriophage lambda that facilitates

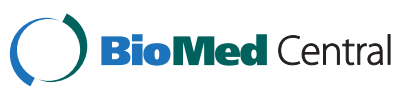




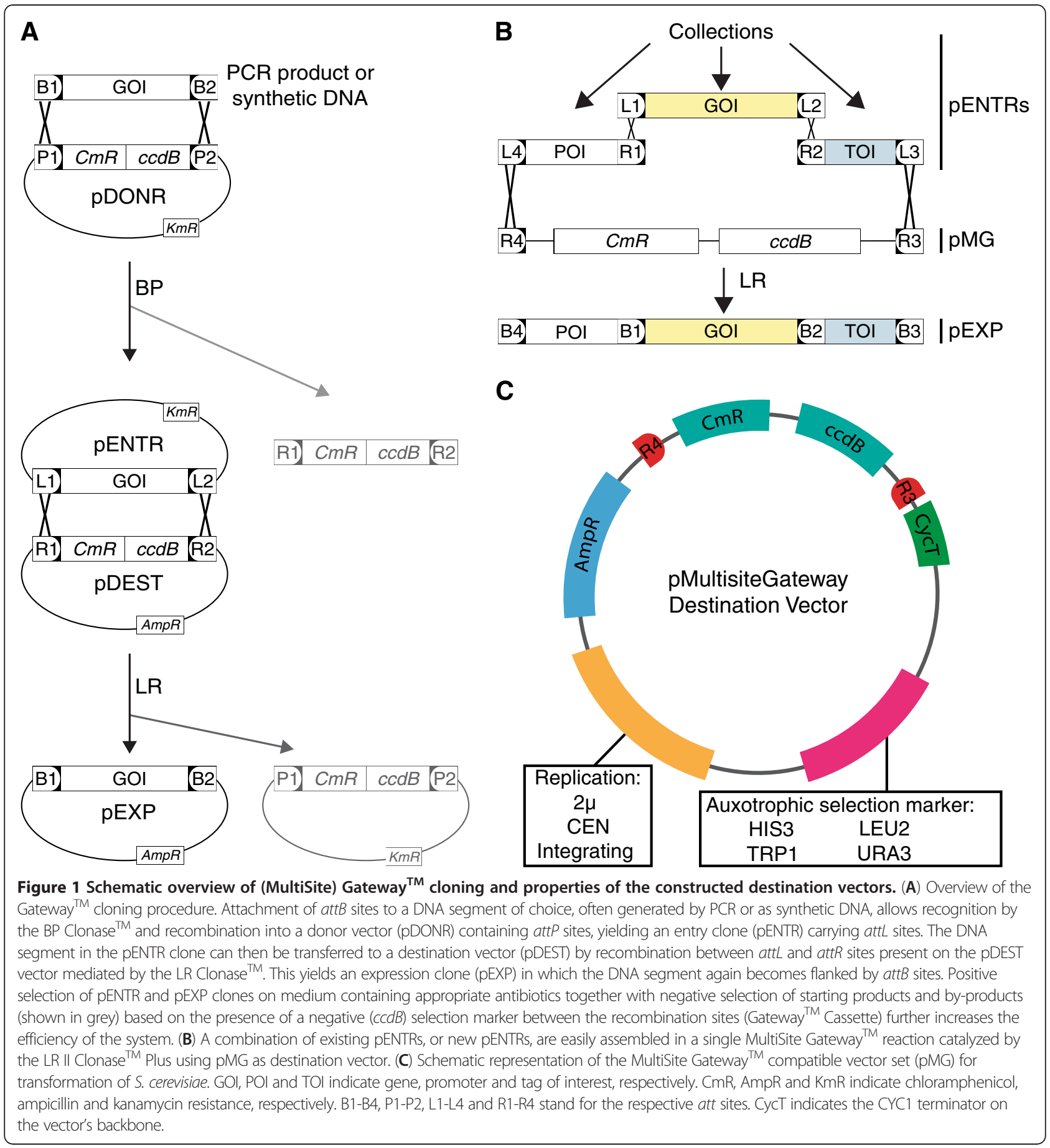

the integration of the phage's DNA into the Escherichia coli chromosome [4]. DNA segments that are flanked by the appropriate recombination sites in a standard vector (pENTR) can easily be transferred to a compatible vector (pDEST) for functional analysis (Figure 1A). MultiSite Gateway $^{\mathrm{TM}}$ uses modified recombination sites to allow the combination of multiple DNA segments in one single in vitro recombination reaction. The segments are joined in a pDEST in a predefined order and orientation, maintaining the reading frame and with low risk for mutations [4] (Figure 1B). Three-segment MultiSite Gateway ${ }^{\mathrm{TM}}$ makes it possible to easily make any combination of a promoter, gene and tag without the need of redesigning new destination vectors for each new experimental approach. The ability to choose a promoter allows varying temporal, spatial, and quantitative control of gene expression, while different possible tags enable the inclusion of fluorescent protein tags for 


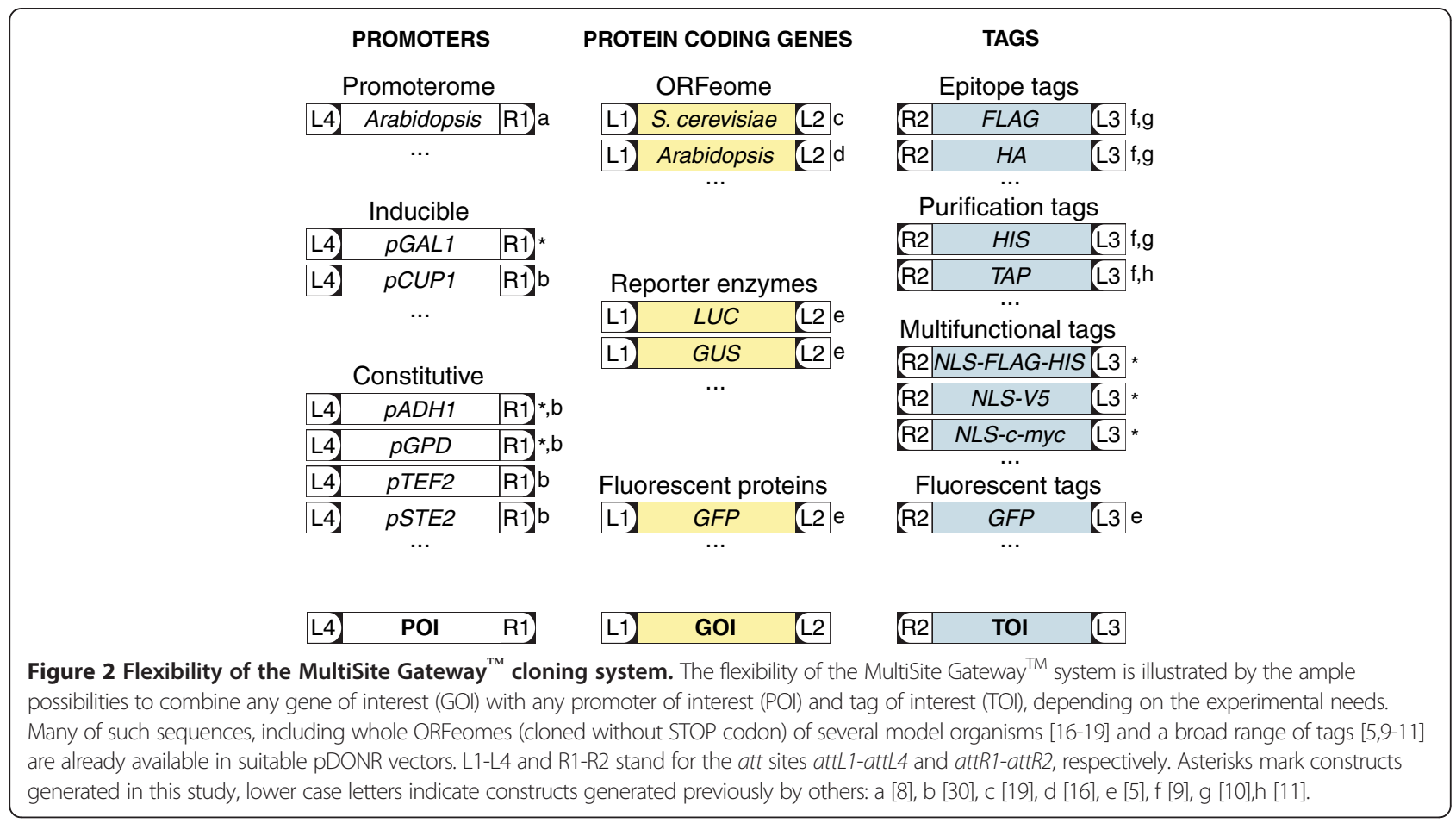

localisation or epitope tags for detection or purification, or the creation of protein chimeras (Figure 2). The flexibility introduced by the MultiSite Gateway ${ }^{\mathrm{TM}}$ technology is illustrated by the large amount of "building blocks" already made available as pENTR clones by several research groups [5-11]. Existing pENTR collections include ORFeomes from multiple prokaryotic and eukaryotic organisms [12-26].

Single-segment pDEST vectors are available for virtually all commonly used systems, such as Drosophila [27]. Moreover, for plants an extensive repertoire of pDEST for two- and three-segment MultiSite Gateway ${ }^{\mathrm{TM}}$ has been established [28]. Three-segment MultiSite Gateway $^{\mathrm{TM}}$ destination vectors are also available for Gram-positive bacteria [29]. In S. cerevisiae, singlesegment pDEST vectors are available for yeast two-hybrid screens (Invitrogen), and two-segment (promoter::ORF) MultiSite Gateway ${ }^{\mathrm{TM}}$ vectors have been described [30]. An extensive set of single-fragment Gateway ${ }^{\mathrm{TM}}$ vectors was constructed, allowing N-terminal fusions with four fluorescent tags, and C-terminal fusions with five different fluorescent tags, an affinity tag and an epitope tag under the control of the inducible GAL1 or constitutive GPD promoter [31]. Alternatively, tags that are not present in this vector set can be fused to the gene of interest through 2-step PCR fusion [32] before performing the $\mathrm{BP}$ reaction. However, to our knowledge no three-segment MultiSite Gateway ${ }^{\mathrm{TM}}$ pDEST vectors exist for $S$. cerevisiae to date. As a consequence, a large number of commonly used protein tags already available as
pENTR clones are not readily applicable in the organism that - together with E. coli - is the workhorse of molecular biology.

In this paper we present a set of eleven functionally validated three-segment MultiSite Gateway ${ }^{\text {TM }}$ pDEST vectors for use in $S$. cerevisiae. The vector set features the four most commonly used auxotrophic markers for selection of yeast transformants combined with three different replication mechanisms. The presence of the CYC1 terminator in these vectors allows construction of any promoter::ORF:tag combination. In addition, a number of useful entry clones harbouring commonly used yeast promoters and entry clones with protein tags to be used with these pDESTs are presented and validated. These vectors have been appended to our collection of 'Gateway ${ }^{\text {TM }}$ vectors for functional studies' and can be ordered through the website http://gateway.psb.ugent.be/. Finally, we illustrate the applicability of this vector set by confirming the formation of a ternary complex between the jasmonate ZIM-domain (JAZ), the Novel Interactor of JAZ (NINJA) and the TOPLESS (TPL) proteins, with NINJA acting as a bridging protein. This complex was previously shown to be involved in jasmonate signalling in the model plant Arabidopsis (Arabidopsis thaliana) [33].

\section{Results and discussion}

Construction of MultiSite Gateway ${ }^{\mathrm{TM}}$ vectors

The MultiSite Gateway ${ }^{\mathrm{TM}}$ cassette of pKm43GW (http://gateway.psb.ugent.be/) flanked by attB4 and 
attB3 sites, was PCR amplified adding XhoI and SacI restriction sites (Additional file 1). The amplification product was subsequently cloned into the XhoI and SacI sites present in the backbones of the vectors of the pAGseries [31]. This step replaced the original Gateway ${ }^{\mathrm{TM}}$ cassette and the eukaryotic promoter from the pAG vectors, while maintaining the $\mathrm{CYC} 1$ terminator. Ligation products were transformed into $c c d B$ resistant $E$. coli cells (One shot $c c d B$ survival $^{\mathrm{TM}}$, Invitrogen). The pAG vectors contain an ampicillin resistance gene for selection in $E$. coli, one of four different auxotrophic selection markers (HIS3, LEU2, TRP1, and URA3) and one of three different replication determinants $(2 \mu$ ori, CEN, or Integrating) thus giving rise to a MultiSite Gateway ${ }^{\mathrm{TM}}$ vector set (Figure 1C) for expression in S. cerevisiae (see Table 1 for nomenclature). We did not succeed in creating the Integrating vector with the HIS3 autotrophic marker (Table 1).

\section{Testing the versatility of the system}

To illustrate the flexibility of this vector collection, we took advantage of a few "building blocks" for MultiSite Gateway $^{\mathrm{TM}}$ cloning that were available in-house as entry clones (Figure 2, http://gateway.psb.ugent.be/). One of the assets of MultiSite Gateway ${ }^{\text {TM }}$ destination vectors is that any promoter of interest can be used, provided it is available as an entry clone flanked by attL4 and attR1 sites. To achieve this, we cloned two constitutively active promoters (pGPD/TDH3 and pADH1) and a galactoseinducible promoter (pGAL1) into pDONR P4-P1R (Invitrogen). The inducibility of the GAL1 promoter was verified by expression in yeast of a heterologous Arabidopsis gene fused to a NLS-FLAG-HIS tag under the

Table 1 Nomenclature of the S. cerevisiae MultiSite Gateway $^{\mathrm{TM}}$ PDEST vectors

\begin{tabular}{lll}
\hline Name* & Replication & Selectable marker \\
\hline pMG304 & Integrating & TRP1 \\
pMG305 & Integrating & LEU2 \\
pMG306 & Integrating & URA3 \\
pMG413 & CEN & HIS3 \\
pMG414 & CEN & TRP1 \\
pMG415 & CEN & LEU2 \\
pMG416 & CEN & URA3 \\
pMG423 & $2 \mu$ & HIS3 \\
pMG424 & $2 \mu$ & TRP1 \\
PMG425 & $2 \mu$ & LEU2 \\
pMG426 & $2 \mu$ & URA3 \\
\hline
\end{tabular}

*pMGXYZ where MG indicates the presence of the MultiSite Gateway ${ }^{\mathrm{TM}}$ cassette; $X$ stands for the presence (4) or absence (3) of replication determinants on the vector; $Y$ stands for the replication determinant: Integrating (0), CEN (1) or $2 \mu$ (2); $Z$ indicates the auxotrophic selection marker: HIS3 (3), TRP1 (4), LEU2 (5) or URA3 (6). control of the GAL1 promoter using pMG416 (Figure 3A).

Another advantage of the MultiSite Gateway ${ }^{\mathrm{TM}}$ system is that several different tag sequences are readily available (Figure 2) and can easily be introduced to acquire translational fusions, since the att sites do not disturb the reading frame. For proof-of-concept, we cloned different plant genes (without STOP codon) in a Cterminal translational fusion with either a V5, c-myc, or FLAG-HIS tag. To make these constructs compatible with protein interaction studies in yeast, we additionally fused each epitope tag with a nuclear localization signal (NLS) derived from SV40, thereby creating the NLS$3 x V 5$, NLS-3xc-myc, and NLS-3xFLAG-6xHIS tags, respectively. Such constructs allow avoiding false negative experimental outcomes that result from protein localisation in different cellular compartments, for instance. Tagged plant proteins were expressed in yeast under the control of the constitutive GPD or ADH1 promoters in different vectors from our set. Total protein extracts were obtained and the expressed proteins were visualized through immunoblot analysis (Figure 3B). The (NLS)FLAG-HIS tag is particularly suitable when protein purification under denaturing conditions is needed, while, at the same time, low background detection on immunoblots is desired.

To express C-terminal translational fusions by means of our promoters, tags and destination vectors, entry vectors containing the GOI should be cloned with a start codon and without a stop codon, and the manufacturer's guidelines regarding the reading frame should be adopted (http://www.invitrogen.com). Organisms for which ORFeome collections contain vectors that match these criteria include several bacterial collections (Brucella melitensis [12], Pseudomonas aeruginosa [13], Bacillus anthracis, Francisella tularensis, Helicobacter pylori, Mycobacterium tuberculosis, Rickettsia prowazekii, Staphylococcus aureus, Streptococcus pneumoniae, Vibrio cholerae [14]), human and mouse [15], plants (Arabidopsis [16], maize, sorghum, sugarcane, rice [17]), viruses [18] and yeasts (S. cerevisiae [19] and Schizosaccharomyces pombe [20]). Caution is advised since some ORFeome collections contain entry clones both with and without stop codon $[15,16]$. ORFeome collections that comprise only GOI's provided with a stop codon, as is the case for some bacterial and viral ORFeomes (Neisseria gonorrhoeae [21], Sinorhizobium meliloti [22], Yersinia pestis [14], and Hepatitis C [23]) are not compatible with our vectors. Other ORFeomes in which the GOI is cloned without a start and without a stop codon (Caenorhabditis elegans [24,25], E. coli [26]), are not compatible with our current promoters but can be used for expression of C-terminal translational fusions with our vectors provided the promoter is cloned followed by 
A

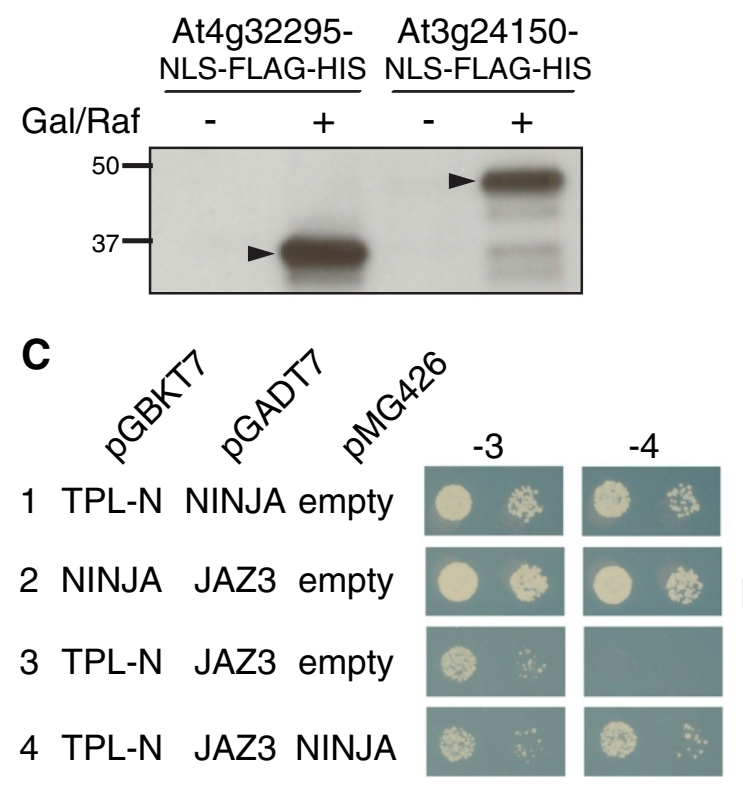

B

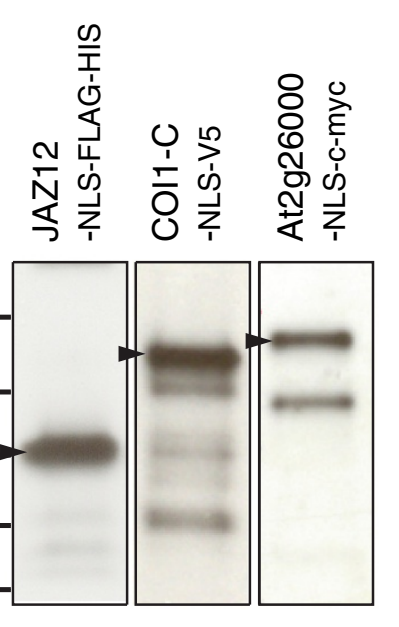

D

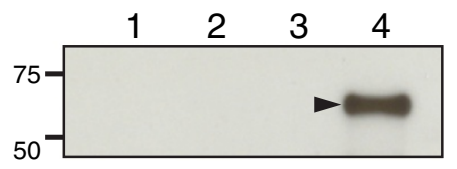

Figure 3 Versatility of the newly generated MultiSite Gateway ${ }^{\mathrm{TM}}$ vector set. (A) Inducibility of the pGAL1 promoter. Arabidopsis genes At4g32295 and At3g24150 were expressed in yeast under the control of the pGAL1 promoter and fused with an NLS-FLAG-HIS tag using the pMG416 vector. After total protein extraction, expression was verified in inducing $(+)$ and non-inducing $(-)$ medium through immunoblot analysis with anti-FLAG antibodies. (B) Functionality of newly developed C-terminal translational fusion epitope tags. Yeasts were transformed with pMG425-ADH1:.JAZ12:-NLSFLAG-HIS, pMG423-GPD::COl1-C:-NLS-V5 where COI1-C is a truncated version of COl1, or pMG423-GPD::At2g26000: NLS-C-myc. Expression of the different constructs was verified with immunoblot analysis of total protein extracts with anti-FLAG, anti-V5 and anti-c-myc antibodies, respectively. (C) Interaction of proteins in the trimeric JAZ3-TPL-NINJA complex in Y3H assays. Transformed yeasts were spotted in 10-fold and 100-fold dilutions on control medium (-3: SD-Leu-Trp-Ura) and selective medium (-4: SD-His-Leu-Trp-Ura). Gene constructs in the pGBKT7 and pGADT7 vectors carry the DNA-binding domain or the transcription activation domain, respectively, in contrast to constructs expressed in pMG426, which do not carry DNA-binding or transcription activation domains. (D) Verification of expression of NINJA: NLS-FLAG-HIS in the Y3H set-ups shown in panel C. After total protein extraction, NINJA: NLSFLAG-HIS was detected through immunoblot analysis with anti-FLAG antibodies. Arrowheads indicate the fusion protein.

a start codon. Since this ORFeome list is non-exhaustive and, as described above, different approaches are used when cloning the GOI, the compatibility of available pENTR collections should always be corroborated either in silico before assembling the different sequences into expression vectors or through epitope tag detection in immunoblots. Finally, the destination vector set presented here also allows expression of $\mathrm{N}$-terminal fusions, provided the att sites flanking the different fusion components are adapted accordingly.

\section{Value of the vector set}

The value of this vector set was exemplified by a yeast three-hybrid $(\mathrm{Y} 3 \mathrm{H})$ experiment, in which we investigated the formation of the ternary protein complex by the Arabidopsis JAZ3, NINJA, and TPL proteins. We have proposed recently that the adaptor protein NINJA bridges the JAZ proteins to TPL proteins and thereby forms a repressor complex that blocks the cellular programs regulated by the jasmonates, ubiquitous plant hormones that regulate various aspects of plant growth, development, and survival [33]. TPL interacts with the
ETHYLENE RESPONSIVE FACTOR-associated amphiphilic repression (EAR) motif that is present in NINJA [33] but absent in most JAZ proteins, including JAZ3. Some JAZ proteins however, i.e. JAZ5 to JAZ8, contain EAR motifs themselves and are capable of direct interaction with TPL [34-36].

The N-terminal domain of TPL contains the LisE and CTHL domains and was previously shown to be essential for binding the EAR motif in Aux/IAA proteins [37]. Therefore we cloned this part (denominated TPL-N) as a bait protein for $\mathrm{Y} 2 \mathrm{H}$. In agreement with the proposed models, the interaction of TPL-N with NINJA was confirmed but TPL-N could not interact with JAZ3 (Figure 3C).

Commonly used $\mathrm{Y} 2 \mathrm{H}$ vectors such as pGADT7 and pGBKT7 (Clontech) are designed such that the bait and prey fusion proteins are targeted to the same subcellular compartment (i.e. nucleus) and are equipped with an epitope tag, HA and c-myc, respectively, allowing easy confirmation of expression through immunoblot. To verify whether NINJA can connect EAR-lacking JAZs with TPL, as previously proposed [33], we performed a 
$\mathrm{Y} 3 \mathrm{H}$ assay in which we expressed NINJA, under control of a constitutive promoter (pGPD), as a bridging protein. Hereby we used the MultiSite Gateway $^{\mathrm{TM}}$ vector pMG426 (Table 1) that carries the URA3 auxotrophic marker that is often still available in yeast strains used for $\mathrm{Y} 2 \mathrm{H}$. As a C-terminal tag we used the NLS-FLAGHIS tag. Only when NINJA is co-expressed, yeast growth was observed on selective -His medium, indicating that interaction between JAZ3 and TPL requires the involvement of NINJA (Figure 3C-D).

\section{Conclusions}

We have successfully constructed a set of three-segment MultiSite Gateway ${ }^{\mathrm{TM}}$ destination vectors for $S$. cerevisiae. Our findings make high-throughput recombinatorial cloning of multiple genetic segments in one single reaction accessible in one of the most widely used experimental model systems in molecular biology. The availability of different auxotrophic markers in this vector set, together with the large amount of existing compatible building blocks for MultiSite Gateway ${ }^{\mathrm{TM}}$ cloning already available in several research groups, creates a versatile utility for these vectors. In addition, we have cloned two constitutive and one inducible yeast promoter in appropriate pENTR vectors and constructed three novel epitope tags, each including a NLS, which are suitable for interaction studies in yeast.

The usefulness of the MultiSite Gateway ${ }^{\mathrm{TM}}$ vectors was demonstrated in a $\mathrm{Y} 3 \mathrm{H}$ assay with which we corroborated the hypothesis that NINJA connects the JAZ proteins with the co-repressor TPL. This trimeric complex mediates repression of jasmonate responsive genes in the absence of the hormone [33].

Implementation of the vector set presented in this article, together with the cloning of more promoters and (epitope) tags according to personal experimental needs, will facilitate gene functional studies and contribute to the high-throughput versatile expression of heterologous (plant) proteins in yeast.

\section{Methods}

\section{Strains and growth conditions}

The E. coli strains used were either the $c c d B$ resistant strain DB3.1 (Invitrogen) or the $c c d B$ sensitive strain $\mathrm{DH} 5 \alpha$. Both were grown at $37^{\circ} \mathrm{C}$ in $\mathrm{LB}$ broth medium with appropriate antibiotics. Several different commonly used yeast lab-strains were grown at $30^{\circ} \mathrm{C}$ in synthetic defined medium (Clontech) lacking the appropriate amino acids.

\section{MultiSite Gateway ${ }^{\mathrm{TM}}$ cloning and yeast transformation}

MultiSite LR reactions were performed in $10 \mu \mathrm{L}$ total volume containing 10 fmoles of each entry vector, 20 fmoles of destination vector, and $2 \mu \mathrm{L}$ LR II Clonase ${ }^{\mathrm{TM}}$
Plus (Invitrogen). The reaction was incubated overnight at $25^{\circ} \mathrm{C}$. After proteinase treatment, the mix was transformed into E. coli DH5 $\alpha$. Colonies that grew on selective medium were picked and the insert was sequenced using M13 forward and reverse primers (Additional file 1). To maintain the reading frame, necessary for expression of translational fusions, MultiSite Gateway ${ }^{\mathrm{TM}}$ cloning was carried out according to the manufacturer's guidelines (http:// www.invitrogen.com). Note that in order to produce C-terminal translational fusions, the ORFs used should be without STOP codon. A convenient method to obtain simultaneously clones of ORFs with and without STOP codon has been described [38].

Competent yeast cells were transformed using the LiAc/SS carrier DNA/PEG method [39].

\section{Promoter cloning}

The $a t t B 4$ and $a t t B 1$ sites were introduced in the primers used for promoter amplification (Additional file 1). A PCR was performed using Phusion High-Fidelity PCR Kit (Thermo Fisher Scientific) on 50 ng of pBEVY-A, pBEVY-GL [40], and pGAD424 (Clontech) as template for the GPD, GAL, and ADH1 promoters, respectively. PCR products were purified with the GeneJET Gel Extraction kit (Fermentas). BP reactions were performed in a total volume of $5 \mu \mathrm{l}$ containing $1 \mu$ l enzyme, $300 \mathrm{ng}$ pDONR P4-P1R (Invitrogen), and 30 ng of PCR product. Incubation and subsequent treatments were the same as those for MultiSite LR reactions.

\section{Epitope-tag design and immunoblot analysis}

Synthetic DNA encoding NLS-3xFLAG-6xHIS, NLS3xV5, and NLS-3xc-myc flanked by attB2R-attB3 sites were designed in Vector $\mathrm{NTI}^{\circledR}$ (Invitrogen) and ordered from GenScript as clones in the pUC57 vector. These tags were introduced into pDONR P2R-P3 through a BP reaction. The resulting entry vectors were transformed into $E$. coli and sequence verified.

Total yeast protein extracts were obtained as described [41] and concentration quantified using the Bio-Rad Protein Assay (Bio-Rad). Samples were combined with $5 \mathrm{x}$ Laemmli loading buffer and denatured for $10 \mathrm{~min}$ at $95^{\circ} \mathrm{C}$. Subsequently, $30 \mu \mathrm{g}$ total protein was loaded on a 4-15\% Mini-PROTEAN ${ }^{\circledR}$ TGX $^{\mathrm{TM}}$ Precast Gel (Bio-Rad) and transferred to a PVDF membrane using the TransBlot Turbo transfer system (Bio-Rad). Detection was performed using the following primary antibodies: antiFLAG (Sigma), anti-c-myc-HRP (Invitrogen), anti-HA (Roche), and anti-V5 (Sigma).

\section{Yeast two- and three-hybrid}

The primers were designed to clone the ORF corresponding to TPL-N with and without STOP codon (Additional file 1) [38]. The entry clones pEN-L4-GPD- 
R1, pEN-R2-NLS-3xFLAG-6xHis-L3, and pEN-L1-NINJA$\mathrm{L} 2$ were recombined by MultiSite Gateway ${ }^{\mathrm{TM}} \mathrm{LR}$ reaction with pMG426 as destination vector. Construction of the pGADT7- and pGBKT7-clones, and the $\mathrm{Y} 2 \mathrm{H}$ and $\mathrm{Y} 3 \mathrm{H}$ were carried out as described [33] except that transformed yeast cells (strain PJ69-4a) were selected on SD-Ura-Trp-Leu.

\section{Additional file}

\section{Additional file 1: Primers used and their sequence.}

\section{Competing interests}

The authors declare that they have no competing interests.

\section{Authors' contributions}

AND, TM and RDC carried out experiments. LP and AG designed experiments. AND and LP wrote the manuscript. All authors read, edited and approved the manuscript.

\section{Acknowledgements}

We thank Mansour Karimi for useful discussion. This work was supported by the Research Foundation-Flanders through the projects GA13111N and G005312N, and a postdoctoral fellowship to LP. This work was supported by the European FP7 project SmartCell (222716).

\section{Author details}

${ }^{1}$ Department of Plant Systems Biology, VIB, Technologiepark 927, B-9052, Gent, Belgium. ${ }^{2}$ Department of Plant Biotechnology and Bioinformatics, Ghent University, Technologiepark 927, B-9052, Ghent, Belgium. ${ }^{3}$ Laboratory of Molecular Cell Biology, Institute of Botany and Microbiology, Katholieke Universiteit Leuven, Kasteelpark Arenberg 31, B-3001, Leuven-Heverlee, Belgium. ${ }^{4}$ Department of Molecular Microbiology, VIB, Kasteelpark Arenberg 31, B-3001, Leuven-Heverlee, Belgium.

Received: 19 March 2012 Accepted: 17 September 2012 Published: 20 September 2012

\section{References}

1. Rual JF, Venkatesan K, Hao T, Hirozane-Kishikawa T, Dricot A, Li N, Berriz GF, Gibbons FD, Dreze M, Ayivi-Guedehoussou N, et al: Towards a proteomescale map of the human protein-protein interaction network. Nature 2005, 437:1173-1178.

2. Deplancke B, Mukhopadhyay A, Ao W, Elewa AM, Grove CA, Martinez NJ, Sequerra R, Doucette-Stamm L, Reece-Hoyes JS, Hope IA, et al: A genecentered C. elegans protein-DNA interaction network. Cell 2006, 125:1193-1205.

3. Ro DK, Paradise EM, Ouellet M, Fisher KJ, Newman KL, Ndungu JM, Ho KA, Eachus RA, Ham TS, Kirby J, et al: Production of the antimalarial drug precursor artemisinic acid in engineered yeast. Nature 2006, 440:940-943.

4. Hartley JL, Temple GF, Brasch MA: DNA cloning using in vitro site-specific recombination. Genome Res 2000, 10:1788-1795.

5. Karimi M, Bleys A, Vanderhaeghen R, Hilson P: Building blocks for plant gene assembly. Plant Physio/ 2007, 145:1183-1191.

6. Petersen LK, Stowers RS: A gateway multisite recombination cloning toolkit. PLoS One 2011, 6:e24531.

7. Kwan KM, Fujimoto E, Grabher C, Mangum BD, Hardy ME, Campbell DS, Parant JM, Yost HJ, Kanki JP, Chien CB: The Tol2kit: a multisite gatewaybased construction kit for Tol2 transposon transgenesis constructs. Developmental dynamics: an official publication of the American Association of Anatomists 2007, 236:3088-3099.

8. Benhamed M, Martin-Magniette ML, Taconnat L, Bitton F, Servet C, De Clercq R, De Meyer B, Buysschaert C, Rombauts S, Villarroel R, et al: Genome-scale Arabidopsis promoter array identifies targets of the histone acetyltransferase GCN5. The Plant journal: for cell and molecular biology 2008, 56:493-504.

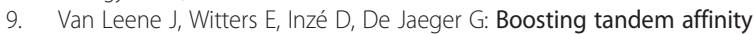
purification of plant protein complexes. Trends in plant science 2008 13:517-520
10. Van Leene J, Stals H, Eeckhout D, Persiau G, Van De Slijke E, Van Isterdael G, De Clercq A, Bonnet E, Laukens K, Remmerie N, et al: A tandem affinity purificationbased technology platform to study the cell cycle interactome in Arabidopsis thaliana. Molecular \& cellular proteomics: MCP 2007, 6:1226-1238.

11. Burckstummer T, Bennett KL, Preradovic A, Schutze G, Hantschel O, SupertiFurga $G$, Bauch A: An efficient tandem affinity purification procedure for interaction proteomics in mammalian cells. Nature methods 2006, 3:1013-1019.

12. Dricot A, Rual JF, Lamesch P, Bertin N, Dupuy D, Hao T, Lambert C, Hallez R, Delroisse JM, Vandenhaute J, et al: Generation of the Brucella melitensis ORFeome version 1.1. Genome Res 2004, 14:2201-2206.

13. Labaer J, Qiu Q, Anumanthan A, Mar W, Zuo D, Murthy TV, Taycher H, Halleck A, Hainsworth E, Lory S, Brizuela L: The Pseudomonas aeruginosa PA01 gene collection. Genome Res 2004, 14:2190-2200.

14. Pathogen functional genomics resource center. http://pfgrc.jcvi.org/.

15. The ORFeome collaboration. http://www.orfeomecollaboration.org/.

16. ATOME 1 and ATOME 2. http://www-urgv.versailles.inra.fr/atome/index.htm.

17. Yilmaz A, Nishiyama MY Jr, Fuentes BG, Souza GM, Janies D, Gray J, Grotewold E: GRASSIUS: a platform for comparative regulatory genomics across the grasses. Plant physiology 2009, 149:171-180.

18. von Brunn A, Teepe C, Simpson JC, Pepperkok R, Friedel CC, Zimmer R, Roberts $R$, Baric R, Haas J: Analysis of intraviral protein-protein interactions of the SARS coronavirus ORFeome. PloS one 2007, 2:e459.

19. Gelperin DM, White MA, Wilkinson ML, Kon Y, Kung LA, Wise KJ, LopezHoyo N, Jiang L, Piccirillo S, Yu H, et al: Biochemical and genetic analysis of the yeast proteome with a movable ORF collection. Genes \& development 2005, 19:2816-2826.

20. Matsuyama A, Arai R, Yashiroda Y, Shirai A, Kamata A, Sekido S, Kobayashi Y, Hashimoto A, Hamamoto M, Hiraoka Y, et al: ORFeome cloning and global analysis of protein localization in the fission yeast Schizosaccharomyces pombe. Nature biotechnology 2006, 24:841-847.

21. Brettin T, Altherr MR, Du Y, Mason RM, Friedrich A, Potter L, Langford C, Keller TJ, Jens J, Howie $H$, et al: Expression capable library for studies of Neisseria gonorrhoeae, version 1.0. BMC microbiology 2005, 5:50.

22. Schroeder BK, House BL, Mortimer MW, Yurgel SN, Maloney SC, Ward KL, Kahn ML: Development of a functional genomics platform for Sinorhizobium meliloti: construction of an ORFeome. Applied and environmental microbiology 2005, 71:5858-5864.

23. de Chassey B, Navratil V, Tafforeau L, Hiet MS, Aublin-Gex A, Agaugue S, Meiffren G, Pradezynski F, Faria BF, Chantier T, et al: Hepatitis $C$ virus infection protein network. Molecular systems biology 2008, 4:230.

24. Lamesch P, Milstein S, Hao T, Rosenberg J, Li N, Sequerra R, Bosak S, Doucette-Stamm L, Vandenhaute J, Hill DE, Vidal M: C. elegans ORFeome version 3.1: increasing the coverage of ORFeome resources with improved gene predictions. Genome research 2004, 14:2064-2069.

25. Reboul J, Vaglio P, Rual JF, Lamesch P, Martinez M, Armstrong CM, Li S, Jacotot $L$, Bertin N, Janky R, et al: $C$. elegans ORFeome version 1.1: experimental verification of the genome annotation and resource for proteome-scale protein expression. Nature genetics 2003, 34:35-41.

26. Rajagopala SV, Yamamoto N, Zweifel AE, Nakamichi T, Huang HK, MendezRios JD, Franca-Koh J, Boorgula MP, Fujita K, Suzuki K, et al: The Escherichia coli K-12 ORFeome: a resource for comparative molecular microbiology. BMC genomics 2010, 11:470.

27. Akbari OS, Oliver D, Eyer K, Pai CY: An Entry/Gateway cloning system for general expression of genes with molecular tags in Drosophila melanogaster. BMC cell biology 2009, 10:8.

28. Karimi M, Depicker A, Hilson P: Recombinational cloning with plant gateway vectors. Plant physiology 2007, 145:1144-1154.

29. Perehinec TM, Qazi SN, Gaddipati SR, Salisbury V, Rees CE, Hill PJ: Construction and evaluation of multisite recombinatorial (Gateway) cloning vectors for Gram-positive bacteria. BMC molecular biology 2007, 8:80.

30. Cheo DL, Titus SA, Byrd DRN, Hartley JL, Temple GF, Brasch MA: Concerted assembly and cloning of multiple DNA segments using in vitro site-specific recombination: functional analysis of multi-segment expression clones. Genome research 2004, 14:2111-2120.

31. Alberti $S$, Gitler $A D$, Lindquist $S$ : A suite of gateway cloning vectors for high-throughput genetic analysis in Saccharomyces cerevisiae. Yeast 2007, 24:913-919.

32. Atanassov II, Etchells JP, Turner SR: A simple, flexible and efficient PCR-fusion/Gateway cloning procedure for gene fusion, site-directed 
mutagenesis, short sequence insertion and domain deletions and swaps. Plant methods 2009, 5:14.

33. Pauwels L, Barbero GF, Geerinck J, Tilleman S, Grunewald W, Perez AC, Chico JM, Vanden Bossche R, Sewell J, Gil E, et al: NINJA connects the co-repressor TOPLESS to jasmonate signalling. Nature 2010, 464:788-791.

34. Causier B, Ashworth M, Guo W, Davies B: The TOPLESS interactome: a framework for gene repression in Arabidopsis. Plant physiology 2012, 158:423-438.

35. Pauwels $L$, Goossens $A:$ The JAZ proteins: a crucial interface in the Jasmonate signaling cascade. The Plant cell 2011, 23:3089-3100.

36. Shyu C, Figueroa P, Depew CL, Cooke TF, Sheard LB, Moreno JE, Katsir L, Zheng N, Browse J, Howe GA: JAZ8 lacks a canonical degron and has an EAR motif that mediates transcriptional repression of jasmonate responses in Arabidopsis. The Plant cell 2012, 24:536-550.

37. Szemenyei $H$, Hannon M, Long JA: TOPLESS mediates auxin-dependent transcriptional repression during Arabidopsis embryogenesis. Science 2008, 319:1384-1386.

38. Underwood BA, Vanderhaeghen $\mathrm{R}$, Whitford $\mathrm{R}$, Town CD, Hilson P: Simultaneous high-throughput recombinational cloning of open reading frames in closed and open configurations. Plant biotechnology journal 2006, 4:317-324.

39. Gietz RD, Schiestl RH: High-efficiency yeast transformation using the LiAc/ SS carrier DNA/PEG method. Nature protocols 2007, 2:31-34.

40. Miller CA 3rd, Martinat MA, Hyman LE: Assessment of aryl hydrocarbon receptor complex interactions using $\mathrm{pBEVY}$ plasmids: expressionvectors with bi-directional promoters for use in Saccharomyces cerevisiae. Nucleic acids research 1998, 26:3577-3583.

41. Hampton RY, Rine J: Regulated degradation of HMG-CoA reductase, an integral membrane protein of the endoplasmic reticulum, in yeast. The Journal of cell biology 1994, 125:299-312.

doi:10.1186/1471-2199-13-30

Cite this article as: Nagels Durand et al: A MultiSite Gateway ${ }^{\mathrm{TM}}$ vector set for the functional analysis of genes in the model Saccharomyces cerevisiae. BMC Molecular Biology 2012 13:30

\section{Submit your next manuscript to BioMed Central and take full advantage of:}

- Convenient online submission

- Thorough peer review

- No space constraints or color figure charges

- Immediate publication on acceptance

- Inclusion in PubMed, CAS, Scopus and Google Scholar

- Research which is freely available for redistribution 